\title{
Economic evaluation of screening programs for hepatitis $C$ virus infection: evidence from literature
}

This article was published in the following Dove Press journal:

Risk Management and Healthcare Policy

2I April 2015

Number of times this article has been viewed

\author{
Silvia Coretti' \\ Federica Romano' \\ Valentina Orlando ${ }^{2}$ \\ Paola Codella' \\ Sabrina Prete' \\ Eugenio Di Brino' \\ Matteo Ruggeri ${ }^{1}$ \\ 'Post-Graduate School of Economics \\ and Management (ALTEMS), \\ Università Cattolica del Sacro \\ Cuore, Rome, Italy; ${ }^{2}$ Center of \\ Pharmacoeconomics (CIRFF), \\ Department of Pharmacy, Federico II \\ University, Naples, Italy
}

Background: Hepatitis $\mathrm{C}$ is a liver infection caused by hepatitis $\mathrm{C}$ virus. Its main complications are cirrhosis and liver cancer. According to the World Health Organization (WHO), more than 185 million people worldwide are infected with hepatitis $C$ virus and, of these, 350,000 die every year. Due to the high disease prevalence and the existence of effective (and expensive) medical treatments able to dramatically change the prognosis, early detection programs can potentially prevent the development of serious chronic conditions, improve health, and save resources.

Objective: To summarize the available evidence on the cost-effectiveness of screening programs for hepatitis $\mathrm{C}$.

Methods: A literature search was performed on PubMed and Scopus search engines. Trip database was queried to identify reports produced by the major Health Technology Assessment (HTA) agencies. Three reviewers dealt with study selection and data extraction blindly.

Results: Ten papers eventually met the inclusion criteria. In studies focusing on asymptomatic cohorts of individuals at general risk the cost/quality adjusted life year of screening programs ranged between US $\$ 4,200$ and $\$ 50,000$ /quality adjusted life year gained, while in those focusing on specific risk factors the incremental cost-effectiveness ratio ranged between $\$ 848$ and $\$ 128,424 /$ quality adjusted life year gained. Age of the target population and disease prevalence were the main cost-effectiveness drivers.

Conclusion: Our results suggest that, especially in the long run, screening programs represent a cost-effective strategy for the management of hepatitis $\mathrm{C}$.

Keywords: hepatitis C, screening, early detection, cost-effectiveness

\section{Introduction}

Hepatitis $\mathrm{C}$ is a liver disease due to the infection with hepatitis $\mathrm{C}$ virus (HCV). The infection is quite often asymptomatic and when symptoms appear they are characterized by muscle pain, nausea, vomiting, fever, abdominal pain, and jaundice. People suffering from hepatitis $\mathrm{C}$ can recover spontaneously with a $20 \%$ probability, but the infection has a high probability $(80 \%-85 \%)$ of becoming chronic, developing as a long-term pathology that may become cirrhosis, a severe pathology that may lead to hepatic impairment and liver cancer. ${ }^{1}$

According to the Guidelines of the World Health Organization (WHO) (April 2014) more than 185 million people worldwide have been infected with the HCV; of these, 350,000 die each year. ${ }^{2,3}$ One-third of those who become chronically infected develop liver cirrhosis or hepatocellular carcinoma (HCC). ${ }^{4}$

$\mathrm{HCV}$ is conventionally classified into seven distinct genotypes (from 1 to 7 ) and a large number of subtypes have been described. ${ }^{5}$ Genotypes and subtypes differ
Correspondence: Silvia Coretti Post-Graduate School of Economics and Management (ALTEMS), Università Cattolica del Sacro Cuore, Largo Francesco Vito I, Rome 00168 , Italy Email silvia.coretti@rm.unicatt.it 
from each other by about $30 \%$ and $20 \%$ of their sequences, respectively. Genotype 1 is the most prevalent worldwide, with a higher proportion of subtype $1 \mathrm{~b}$ in Europe and 1a in the US. Genotype 3a is highly prevalent in the European population among injecting drug users (IDUs). However, such a population is currently experiencing an increasing incidence and prevalence of infections with HCV genotype 4. Genotype 2 is found in clusters in the Mediterranean region, while genotypes 5 and 6 are rare in Europe. ${ }^{6}$ The novel genotype 7 has been identified in patients from Canada and Belgium, possibly infected in Central Africa. ${ }^{7}$

HCV transmission through blood or blood-related products is the most common. However, in developed countries post-transfusion hepatitis $\mathrm{C}$ has become relatively rare due to the spread of procedures of donor screening. Conversely, in developing countries, blood products still represent the main source of transmission. Moreover, in developed countries, $\mathrm{HCV}$ is mainly transmitted by drug abusers sharing injection equipment, with prevalence of anti-HCV among intravenous drug users ranging from $31 \%$ to $98 \%$ across countries. Unsafe therapeutic injections are another important source of transmission in developing countries. Other, but less frequent means of $\mathrm{HCV}$ transmission are tattooing, acupuncture, sharing cottons, body-piercing, cosmetic procedures, and commercial barbering. Finally, vertical transmission from mother to neonate is becoming increasingly rare. ${ }^{8}$

Several medicines are currently available for the treatment of $\mathrm{HCV}$, and healing rates have been steadily improving since the introduction of the latest molecules. The field of HCV therapeutics is evolving rapidly, and a number of compounds are at various stages of their development. These can cure more than $90 \%$ of patients infected with HCV and are effective against genotypes that were previously difficult to treat. Currently, licensed treatments for HCV infection include pegylated (PEG) and standard IFN alpha, ribavirin (RBV), the protease inhibitors boceprevir, telaprevir, and simeprevir; and the NS5B nucleotide polymerase inhibitor sofosbuvir.

The WHO Guidelines Development Group recommends the currently approved direct-acting antiviral molecules (ie, telaprevir, boceprevir, or simeprevir), in combination with PEG-IFN and RBV for patients with genotype $1 \mathrm{HCV}$ infection rather than the dual regimen including only PEG-IFN and RBV. Therefore, genotyping is indicated prior to selecting the appropriate regimen. ${ }^{9}$

In addition, simeprevir, in combination with PEG-IFN and $\mathrm{RBV}$, is recommended for patients with genotype $1 \mathrm{~b}$
HCV infection and for those with genotype $1 \mathrm{a} \mathrm{HCV}$ infection without the Q80K polymorphism, rather than dual treatment with PEG-IFN and RBV alone.

Finally, sofosbuvir and RBV with or without PEG-IFN, depending on the genotype, is recommended for patients with genotypes $1,2,3$, or $4 \mathrm{HCV}$ infection. ${ }^{9}$

Nonetheless, the high cost of these innovative medical treatments, along with the increasing pressures for the containment of health care expenditure, especially in developed countries, suggest that policy makers reconsider the role of prevention and the early detection in the management of hepatitis $\mathrm{C}$.

However, no vaccine for $\mathrm{HCV}$ prevention is available yet, due to its ability to quickly mutate eluding the immune system. However, asymptomatic patients can be identified through screening programs. In this phase, patients identified with early diagnosis have higher probability to be efficiently (and effectively) treated and healed.

Screening for $\mathrm{HCV}$ infection requires an initial serologic screening test followed by an HCV ribonucleic acid (RNA) test to confirm the presence of viremia, and therefore chronic infection, as $15 \%-45 \%$ of patients initially infected will spontaneously clear the virus usually within 6 months of acquiring the infection. People who do not clear HCV within 6 months definitely have chronic HCV infection and are diagnosed either during routine screening or when they develop symptoms of HCV-associated liver disease. ${ }^{10,11}$

If adequately designed, early detection strategies might ensure prompt treatment of infected patients and allow better targeting of the new effective, but also costly, medical treatments as suggested by recent studies investigating the cost-effectiveness of the new molecules. ${ }^{12,13}$ This would ultimately reduce the occurrence of chronicity-related complications with a twofold positive impact on both patients' health and health care expenditure.

This study aims to summarize the available evidence on the cost-effectiveness of screening programs for $\mathrm{HCV}$.

\section{Methods}

\section{Search strategy}

A systematic literature review has been conducted querying both PubMed and Scopus search engines using the following keywords: HCV screening, cost-effectiveness analysis, cost-utility analysis, cost-benefit analysis, cost minimization analysis, economic evaluation, economic assessment, and economic burden. Boolean operators were 
utilized to link the keywords to each other. The search was limited to studies published in the last 10 years in English language.

Moreover, TRIP database was utilized to identify further economic assessments and reports or guidelines produced by the main Health Technology Assessment agencies worldwide, potentially useful as sources of background information.

\section{Inclusion/exclusion criteria}

In order to be included in the analysis, the records retrieved had to fulfill the following inclusion criteria jointly:

- study type: full economic evaluation, entailing the comparison of two or more health care programs in terms of both costs and consequences;

- study design: economic assessment performed alongside randomized controlled trials, observational studies, natural experiments, or secondary analyses carried out through decision models;

- population: asymptomatic population at general or high risk of infection with $\mathrm{HCV}$;

- intervention: hepatitis $\mathrm{C}$ screening program;

- comparator: no early detection measure and treatment of symptomatic patients solely; screening for hepatitis $\mathrm{C}$ with a different scheme (eg, two different age cohorts compared or targeted versus mass screening);

- outcome: outcome of the economic evaluation expressed in terms of cost/quality adjusted life year (QALY) gained or cost/unit of effectiveness gained (eg, life year [LY], cost per case detected).

Thus, partial economic evaluations (ie, analysis focusing on either costs or consequences of screening programs), reviews, pilot studies, and editorials were considered not eligible for this review. Moreover, economic evaluations concerning pre-donation screening of hepatitis $\mathrm{C}$ were not taken into account.

\section{Review process and data extraction}

The studies identified were assigned to two reviewers (FR, PC) who performed a preliminary screening of titles and abstracts based on the mentioned inclusion/exclusion criteria. Potentially relevant studies were then reviewed considering the full text through a blinded process. Several pieces of information concerning the features of included studies, as well as their results, were extracted namely the study setting, the analysis perspective, the analysis time horizon, the discount rate, the study type, the study popula- tion, the alternatives compared, the cost items considered in the analysis, base case results and, lastly, the results of the sensitivity analysis. Any disagreement between the two reviewers was solved by a senior researcher (SC). Results from the different studies were finally compared and discussed qualitatively.

\section{Results}

The PubMed search yielded 147 records, while searching Scopus identified 57 records. Of these, two were duplicates and three were not available. Of the remaining 199 records, 16 were excluded based on title and abstract reading for lack of relevance. Finally, 183 full texts were reviewed. After reading them, ten papers were included in this analysis. The remaining articles were excluded because:

1. they were not relevant to the purpose of the study (87);

2. costs analysis was not available (38);

3. they were literature reviews (48).

The algorithm in Figure 1 depicts this selection phase.

\section{General description of the included studies}

The ten articles included in this research reported full economic evaluations of the HCV screening programs. In particular, most of them (eight) were cost-utility analyses reporting results in terms of cost per QALYs gained; one study was a cost-effectiveness analysis showing results in terms of cost per LY gained, and finally, in one study the

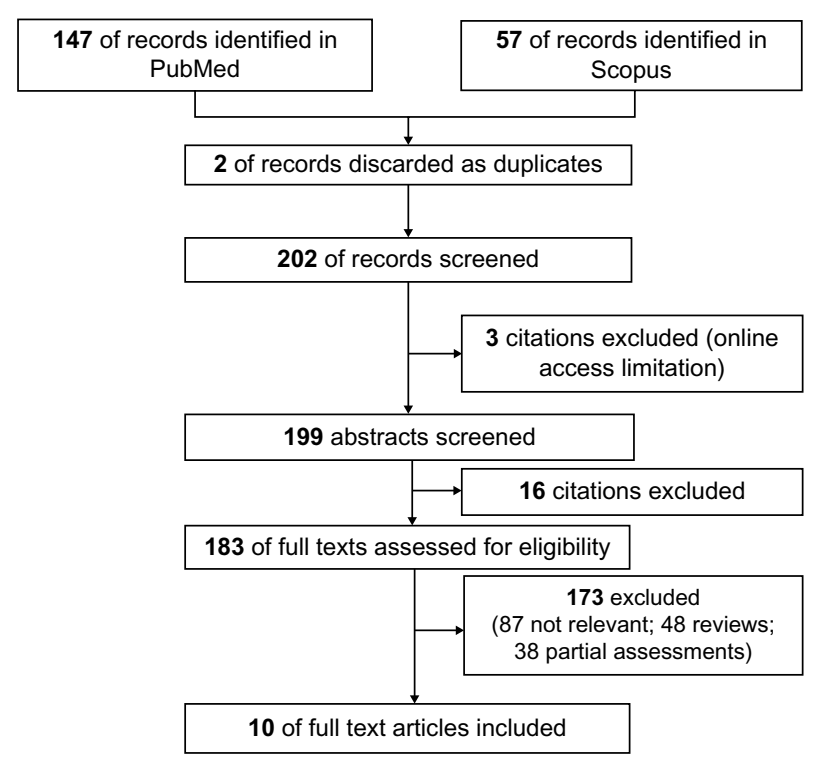

Figure I Flowchart of the study selection process. 
authors carried out both cost-utility and cost-effectiveness analyses. ${ }^{14,15}$

In most of the studies selected (nine), a Markov model was constructed, while Cipriano et al developed a deterministic dynamic compartmental model. ${ }^{15}$

The time horizon of the models included in the analyses was lifetime. Only one study reported a different time horizon ( 80 years), to keep in account the peculiarity of the model population. ${ }^{16}$

The population of these studies was represented by asymptomatic patients already suffering from other hepatic diseases, asymptomatic patients, pregnant women, asymptomatic patients with risk factors, IDUs, and prisoners. Half of the papers (five) report an analysis considering the general population as a target for the screening program, while the others focus solely on high-risk sub-populations.

The studies, conducted between 2008 and 2013, were performed in different countries: five in the US, one in the UK, one in the Netherlands, one in Japan, and two in Italy. The screening programs assessed in these studies were mass screenings, or screenings addressed to specific subpopulations identified by risk factors, age cohort, state of pregnancy, injection drug use, detention in prison. In most of the studies, screening programs were associated with the medical treatment of positive patients and compared to the treatment of symptomatic patients only. More in detail, medical treatment strategies taken into account were: triple therapy (telaprevir/boceprevir-based regimens), dual therapy with PEG-IFN and RBV, IL28B triple therapy.

Most of these studies (six) adopted the third party payer perspective (ie, National Health Service, Health care system, private insurer). In these analyses, only direct costs related to either the disease or to the screening program or both were considered. In the remaining four studies the societal perspective was chosen.

Studies included in the present analysis evaluated resource consumption associated with interventions and comparators by considering different items of direct medical costs, namely costs of HCV health states (chronic hepatitis, compensated and decompensated cirrhosis, HCC, liver transplant), screening costs, drugs costs, laboratory testing, office visit costs, and hospitalizations.

In most of the studies, costs and consequences were discounted at the same rate (either $3 \%$ or $3.5 \%$ annual rate). Urbanus et al, instead, took into account a $4.5 \%$ annual rate for costs and a $1.5 \%$ annual rate for consequences. ${ }^{14}$

In order to test the robustness of results, most of the studies included a deterministic sensitivity analysis which was one-way in most of the cases. Six papers included a probabilistic sensitivity analysis as well.

Further detail on the cost-effectiveness estimates from studies included in the current review is provided in the following section.

\section{Cost-effectiveness of screening programs for hepatitis $C$}

Eckman et al developed a Markov model to appraise the cost-effectiveness of a screening program addressed to the American general population compared to no early detection strategy. Telaprevir/boceprevir-based regimens were assumed as medical treatments for HCV. The screening program exhibited an incremental cost-effectiveness ratio (ICER) of \$47,276/QALY when associated with boceprevir-based regimen, and \$44,074/QALY when associated with telaprevirbased regimen. Model results proved to be sensitive to $\mathrm{HCV}$ prevalence, with screening program not being cost-effective when HCV prevalence is lower than $0.84 \% .{ }^{17}$

Liu et al also used a Markov model. Nine screening strategies combined with various treatment options were compared: no-screening, screening for risk factors, screening for birth cohort, standard therapy with IFN and RBV, IL28B, triple standard therapy with an inhibitor or triple universal therapy. All incremental costs and QALYs were compared to the strategy "no screening + standard therapy". Positive, yet non-favorable ICERs referred to birth cohort screening + triple universal therapy $(\$ 65,749 / \mathrm{QALY})$, cohort screening + IL28B treatment $(\$ 60,590 /$ QALY), no screening + IL28B (\$50,417/QALY). The birth cohort screening was more cost-effective than the screening for risk factors. Cost-effectiveness was sensitive to fibrosis-stage and age of individuals. The scenario analysis revealed that birth cohort screening followed by universal triple therapy was the optimal strategy for 40- to 64-year-old individuals with a cost ranging between $\$ 30,000$ and $\$ 50,000$ per QALY gained. ${ }^{18}$

Ruggeri et al developed a Markov model comparing a screening program for the exposed population followed by treatment of individuals testing positive, to a second arm representing the treatment of patients with cirrhosis or HCC. PEG-IFN alfa2a and PEG-IFN alfa2b in combination with RBV were assumed as medical treatments. The screening program had an ICER of $€ 5,171 /$ QALY, which is far below the acceptability threshold of $£ 20,000-30,000 /$ QALY. Results were sensitive to the age of the target population, the prevalence of $\mathrm{HCV}$ infection, and the time horizon adopted. ${ }^{19}$

Urbanus et al focused on a cohort of migrant and nonmigrant pregnant women comparing screening and no 
screening programs. The incremental cost per screened nonmigrant woman was $€ 41$, while the cost per QALY gained was $€ 52,473$, greater than the hypothesized threshold of $€ 50,000$ / QALY. Regarding screening for migrant pregnant women, the ICER was $€ 47,113 /$ QALY: in this case, the screening was more cost-effective than programs for non-migrant pregnant women. The Dutch study demonstrated that both the screening programs were not cost-effective. Probabilistic sensitivity analysis corroborated the base-case results since only $10 \%$ of simulations were cost-effective. ${ }^{14}$

Cipriano et al estimated the cost-effectiveness of HIV and HCV screening on IDUs in opioid replacement therapy (ORT). They evaluated one-time and repeated screening at intervals ranging from annually to once every 3 months. The ICER of each strategy was calculated comparing it to the next-best alternative. Adding HIV and HCV viral RNA testing to antibody testing averted 14.8-30.3 HIV and 3.7-7.7 HCV infections in a screened population of 26,100 IDUs entering ORT over 20 years, depending on screening frequency. Screening every 6 months for HIV antibodies and RNA, and for HCV antibodies upon entry to ORT cost $\$ 57,200 /$ LY gained and it was a dominating strategy in terms of cost per QALY; further increasing the frequency of HCV antibody screening increased the cost to $\$ 71,400$ / LY gained. Screening every 3 months for HIV antibodies and RNA and annually for HCV antibodies cost $\$ 100,750$ / LY gained. Including HCV antibody testing upon entry to ORT increased the ICER to \$168,600/QALY. Strategies including $\mathrm{HCV}$ testing had ICERs exceeding $\$ 100,000$ / QALY gained. The cost-effectiveness of HCV screening strategies improved when awareness of HCV-positive status was associated with a reduction in needle-sharing behavior and was not associated with a decrement in quality of life. Although annual screening was moderately cost-effective compared to no screening, this strategy was less effective and more costly than strategies that include more frequent HIV screening. ${ }^{15}$ Coffin et al compared costs and effectiveness of screening plus therapy in 20- to 69-year-old population versus screening for risk factors, within the American setting. In the first strategy, enrolling $15 \%$ of the population would produce an ICER of \$7,900/QALY, while enrolling $60 \%$ of the population would produce an ICER of $\$ 10,900 /$ QALY. Moreover the screening program exhibited an ICER of $\$ 4,200 /$ QALY if addressed to individuals born between 1945 and 1965. The latter strategy was more cost-effective compared to screening for risk factors and screening for the general population. Not surprisingly, the deterministic sensitivity analysis showed that the ICER increased in infected people not presenting fibrosis, and decreased in those presenting fibrosis. ${ }^{20}$

McGarry et al also carried out a cost-utility analysis of screening programs for American asymptomatic population born between 1946 and 1970 compared to screening programs for risk factors. Again, the screening program for birth cohort was cost-effective, showing an ICER of $\$ 37,700$ / QALY compared to the screening for risk factors. ${ }^{21}$

Nakamura et al evaluated cost and effectiveness of screening programs associated with dual medical treatment (PEGIFN + RBV) in symptomatic patients in Japan and people with high risk factors compared to no screening strategy. The screening program encompassed HCV antibodies test, antigen test, and an $\mathrm{HCV}$-polymerase chain reaction test. The screening in the general population was more expensive than no screening strategy but led to an increase in quality adjusted life expectancy. Thus, the ICER for screening in general population ranged between $\$ 848$ and $\$ 4,825 /$ QALY while the ICER for high-risk population ranged between $\$ 749$ and $\$ 2,297 /$ QALY. In both the programs, the screening was costeffective if compared with no screening strategy. ${ }^{22}$ The model utilized by Sutton et al followed two hypothetical cohorts of individuals. Case-finding strategy, whereby individuals were offered testing and treatment on reception into prison with the chance of spontaneous screening and treatment, was compared to no case-finding arm, in which individuals were only provided with the opportunity of spontaneous screening and treatment. Taking parameter at baseline values, the analysis suggested that HCV screening and treatment in a prison setting was not cost-effective with an estimated cost/ QALY gained of $£ 54,852$. However, reducing the case-finding costs and increasing treatment acceptance and adherence would make that the more cost-effective intervention, and therefore acceptable to the health care payer. ${ }^{16}$

Tramarin et al estimated the cost-effectiveness of HCV screening in two cohorts: IDUs and individuals who had undergone surgery using primary data. In the IDU cohort, the incremental analysis showed that screening was the dominant strategy compared to the expected costs and quality adjusted survival of those patients if they had not undergone screening. Similar findings were observed in the subgroup of patients with genotypes 1 and 4, while in the subgroup of patients with genotypes 2 and 3, the incremental cost per QALY of screening over no screening was $€ 9,659$. In the individuals with surgery cohort, the incremental cost per QALY gained with screening was $€ 918,147$ (€699,991 in the subgroup of genotypes 1 and 4; $€ 2,324,471$ in the subgroup of genotypes 2 and 3 ). The sensitivity analysis confirmed the base-case 


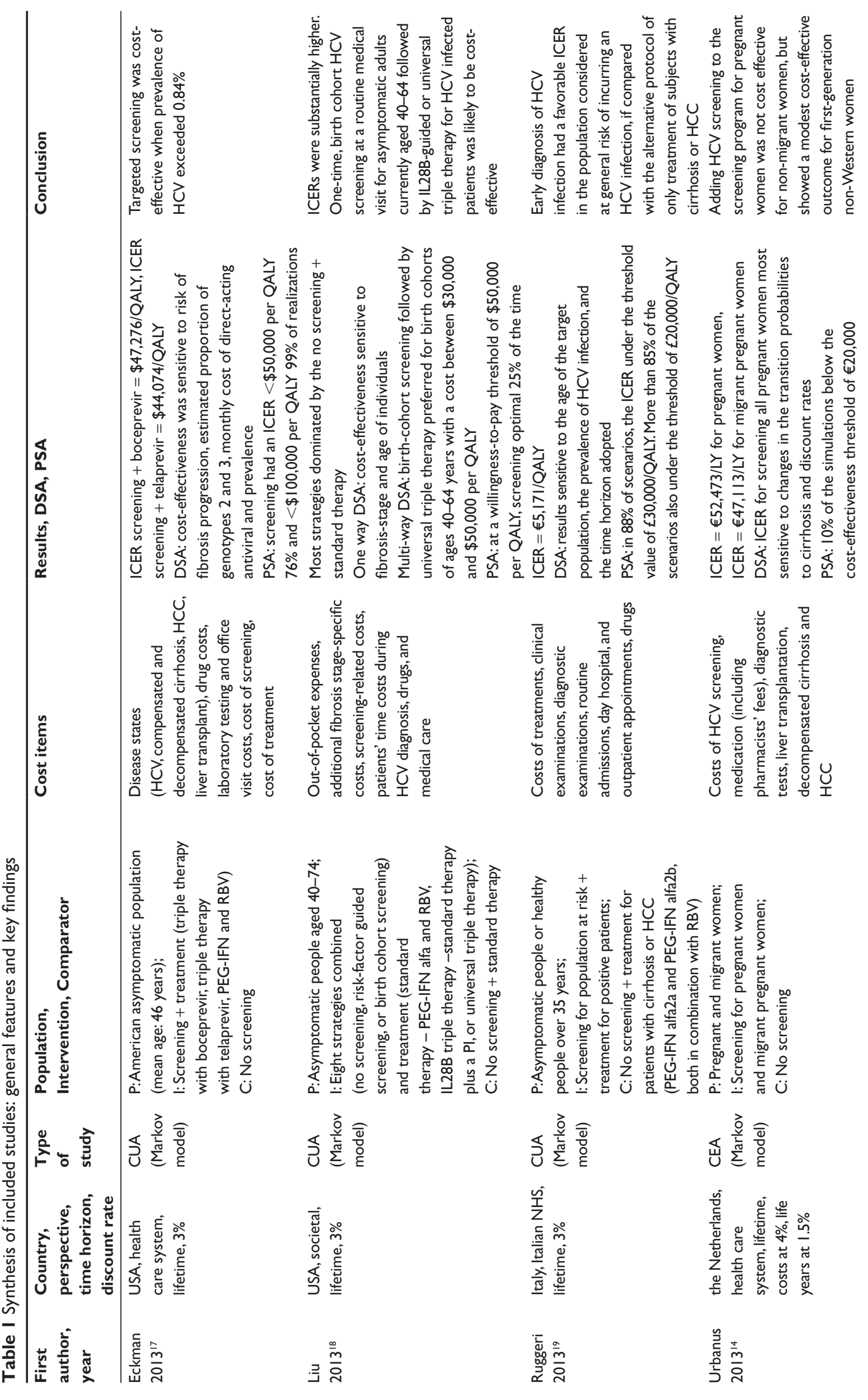



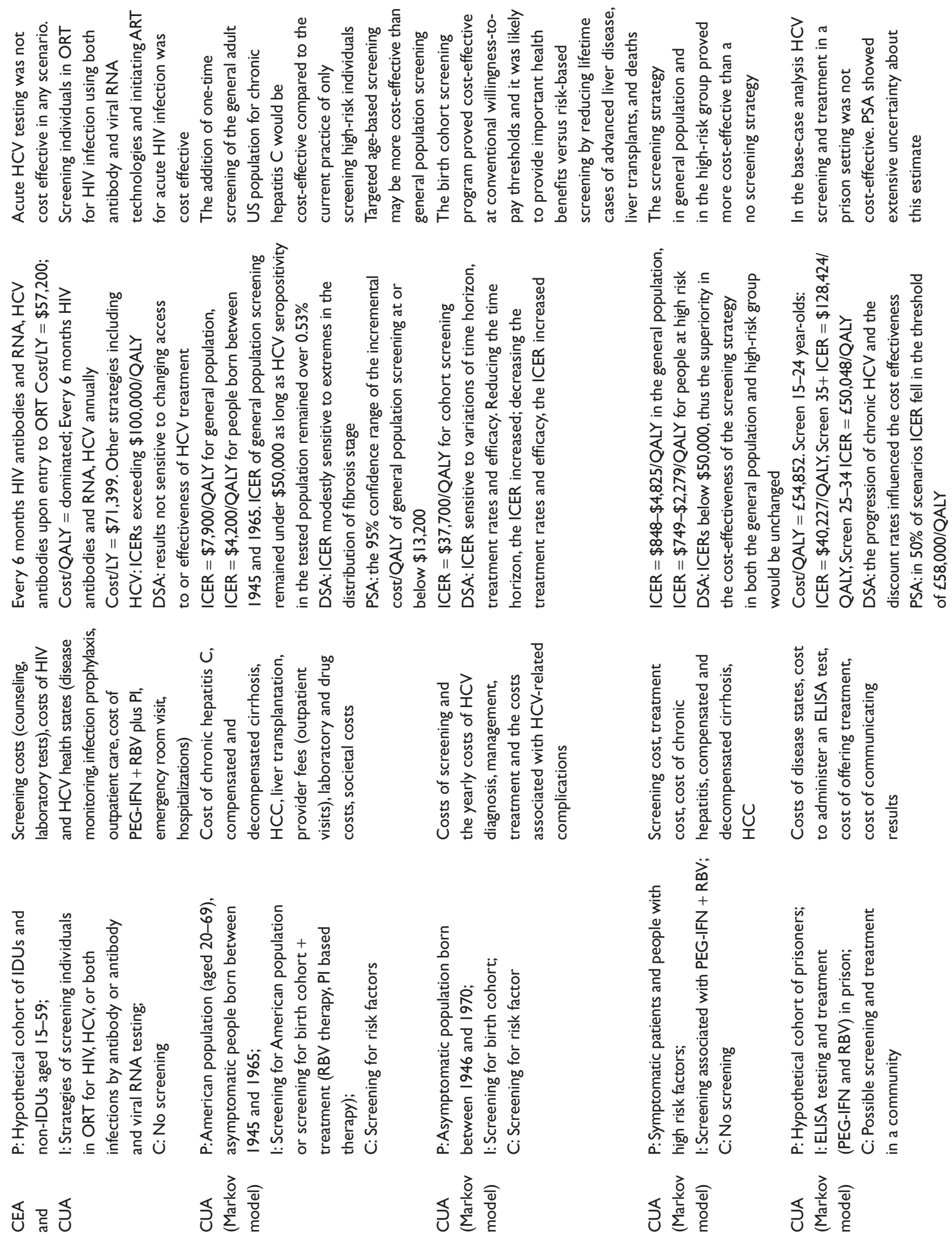

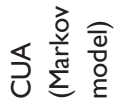

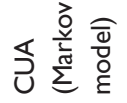

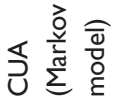

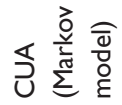

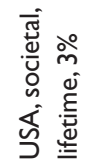

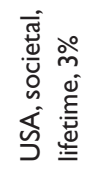

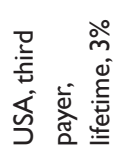

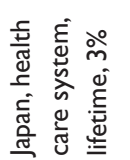

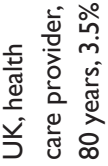

ำ

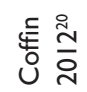

这

己

苍 웅 


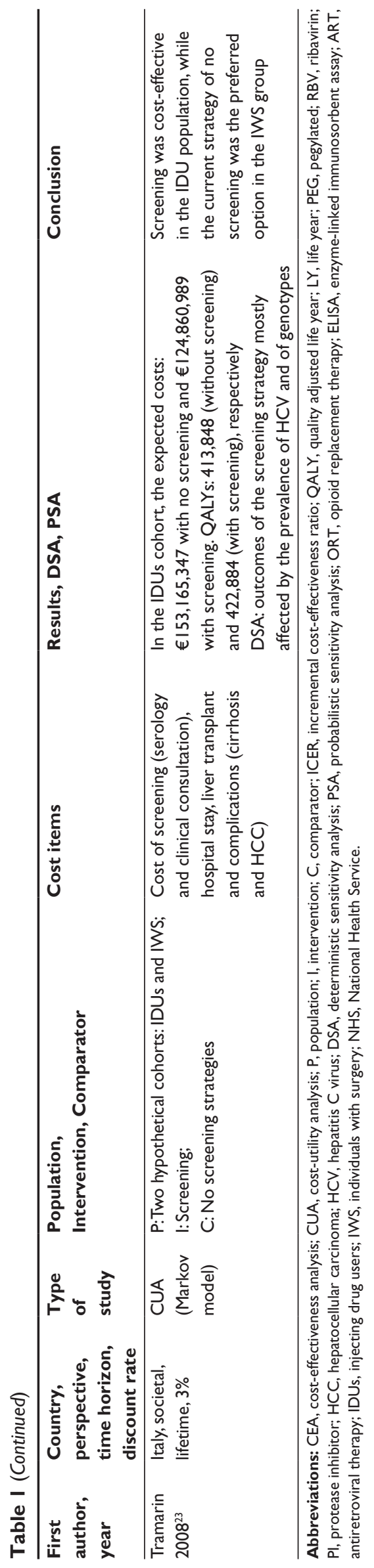

finding, suggesting that screening was not cost-effective in the individuals with surgery cohort even when favorable assumptions were made. In the IDU cohort, instead, screening was the preferred option at a prevalence of $10 \%$ or higher of genotypes 1 and $4 .{ }^{23}$

Table 1 summarizes the main features and findings of the studies included in the current research.

\section{Discussion and conclusion}

In a context of increasing health care needs and ever stringent budget constraints, preventive care and early diagnosis are of paramount importance to safeguard universal access and improve patients' prognosis. Generally, the earlier the treatment starts, the greater the possibility for a full recovery and, in this sense, screening programs are quite often very useful in defining and anticipating diseases and their possible complications. Thus, awareness of the expected costs and benefit of early detection programs can be useful in supporting rational resource allocation decisions. This is also the case of HCV for which new molecules can cure more than $90 \%$ of patients and be effective also for genotypes that were previously difficult to treat. On the other hand, the high cost of these innovative treatments, along with the increasing pressures for cost containment, suggest that policy makers reconsider the role of early detection in the management of hepatitis $\mathrm{C}$.

The aim of this review was to assess the cost-effectiveness of screening programs for hepatitis C. Ten papers were reviewed in the current analysis. Studies included in the analysis considered different interventions, different comparators and populations, and this makes the comparison of results very tricky. Moreover, since the selected studies have been conducted in different contexts and under different perspectives, threshold values adopted for cost-effectiveness are also very heterogeneous. Nonetheless, it is possible to draw some general conclusions.

In studies focusing on asymptomatic cohorts of individuals at general risk the cost/QALY of screening programs ranged between $\$ 4,200$ and $\$ 50,000 / \mathrm{QALY}$ gained. These studies generally proved the cost-effectiveness of screening programs for specific age cohorts in which the disease prevalence is high and life expectancy is sufficiently long. Also, among studies focusing on cohorts characterized by specific risk factors (eg, IDUs, migrant women, prisoners), age proved to play a central role in determining the cost-effectiveness of the early detection strategy. For example Sutton et al, comparing different screening strategies for prisoners, found that early detection exhibited the most favorable ICER in individuals aged $25-34 .{ }^{16}$ This is not surprising, since HCV 
infection may remain asymptomatic for a long time and individuals can develop chronic liver disease after decades. For this reason, the value of early detection increases when life expectancy is sufficiently long.

Since triple therapy has been introduced in clinical practice, healing rates have been steadily improving. Even if the new molecules are more expensive than the standard of care, represented by PEG-IFN + RBV, they dramatically improve the patient's prognosis in terms of survival and quality of life and are effective also in patients with severe liver diseases. This is somehow reflected by our results, in which the ICER of early detection strategies tend to be higher (and thus less favorable) when screening programs are compared to treatment strategies including the new molecules. However, such a finding needs to be interpreted with caution, due to the limited number and the scarce comparability of the studies included in the current analysis. A specific analysis would be needed to address this issue more systematically.

We did not find remarkable differences, in terms of ICER, relating to the study perspective adopted in the selected studies. In particular, we expected to find lower ICERs for screening programs when the analysis was carried out under the societal perspective, due to the high incidence of indirect costs in patients affected by severe liver conditions. This is not always the case in the studies included but, again, since the included studies have been conducted in different countries with diverse models of health care and welfare systems it is not possible to draw definite conclusions on this aspect.

Our review exhibits two main limitations. Firstly, the inclusion criteria for study selection did not consider the methodological quality or the quality of reporting of the records identified. On the other hand, all the selected studies are published on indexed and peer reviewed journals, and using additional inclusion criteria would have meant further reducing the number of studies included. Secondly, our review focused on screening strategies for HCV but did not consider the cost-effectiveness of the specific tests that can be used for early detection. However, during the study selection process two interesting contributions on this topic were identified. Liu et al showed that FibroTest Only was less costly and more effective than liver biopsy only. When they considered triple therapy as medical treatment for HCV, FibroTest Only was cost-effective with an ICER of \$21,200/QALY for men and \$26,100/QALY for women compared to FibroTest Rule In. ${ }^{24}$ Canavan et al showed that with an acceptability threshold of $£ 30,000 /$ ALY gained, annual definitive FibroScan was the optimal strategy. The ICER for annual FibroScan was $£ 6,557.06$ /QALY gained over the next best strategy: symptomatic investigation and treatment with no fibrosis surveillance or HCC screening. Definitive FibroScan replacing biopsy in current surveillance was an extendedly dominating strategy; all other strategies were strictly dominated. The authors concluded that FibroScan should be rationed to replace biopsy in current practice if its cost ranges between $£ 164$ and $£ 658$ per procedure. ${ }^{25}$

In conclusion, our results show that screening programs for hepatitis $\mathrm{C}$ could represent a viable and cost-effective strategy for a more rational allocation of health care resources within this clinical area. However, in order to be cost-effective, screening programs should be targeted to specific sub-populations (eg, particular birth cohorts or high-risk sub-populations) characterized by high disease prevalence. Further studies might reveal which are the most effective and cost-effective organizational arrangements for screening programs, as well as the most appropriate target population.

\section{Disclosure}

The authors declare no conflicts of interest.

\section{References}

1. European Association for the Study of the Liver. EASL Clinical Practice Guidelines: management of hepatitis C virus infection. J Hepatol. 2011; 55(2):245-264.

2. Mohd Hanafiah K, Groeger J, Flaxman AD, Wiersma ST. Global epidemiology of Hepatitis $\mathrm{C}$ virus infection: new estimates of agespecific antibody to HCV seroprevalence. Hepatology. 2013;57(4): 1333-1342.

3. Lavanchy D. The global burden of Hepatitis C. Liver Int. 2009; 29 Suppl 1:74-81.

4. Ly KN, Xing J, Klevens RM, Jiles RB, Ward JW, Holmberg SD. The increasing burden of mortality from viral hepatitis in the United States between 1999 and 2007. Ann Intern Med. 2012;156(4): 271-278.

5. Smith DB, Bukh J, Kuiken C, et al. Expanded classification of hepatitis C Virus into 7 genotypes and 67 Subtypes: updated criteria and assignment web resource. Hepatology. 2014;59(1):318-327.

6. Antaki N, Craxi A, Kamal S, et al. The neglected hepatitis C virus genotypes 4, 5, and 6: an International consensus report. Liver Int. 2010;30(3):342-355.

7. Murphy D, Chamberland J, Dandavino R, Sablon E. A new genotype of hepatitis C virus originating from central Africa. Hepatology 2007;46: 623A.

8. Lee MH, Yang HI, Yuan Y, L'Italien G, Chen CJ. Epidemiology and natural history of hepatitis C virus infection. World $J$ Gastroenterol. 2014;20(28):9270-9280.

9. World Health Organization. Guidelines for the screening, care and treatment of persons with hepatitis C Infection. World Health Organization; 2014.

10. Alter MJ, Seeff LB, Bacon BR, Thomas DL, Rigsby MO, Di Bisceglie AM. Testing for hepatitis $C$ virus infection should be routine for persons at increased risk for infection. Ann Intern Med. 2004;141(9):715-717.

11. Ghany MG, Strader DB, Thomas DL, Seeff LB; American Association for the Study of Liver Diseases. Diagnosis, management, and treatment of hepatitis C: an update. Hepatology. 2009;49(4):1335-1374. 
12. Iannazzo S, Colombatto P, Ricco G, Oliveri F, Bonino F, Brunetto MR. A cost-effectiveness model to personalize antiviral therapy in naive patients with genotype 1 chronic hepatitis C. Dig Liver Dis. 2015;47(3): 249-254.

13. Ruggeri M, Cicchetti $\mathrm{A}$, Coretti S, Cammà C, Caporaso N, Gasbarrini A. Il modello WEF per la valutazione economica delle innovazioni terapeutiche in epatologia. [A new decision model for economic evaluation of novel therapies for HCV]. Farmeconomia Health economics and therapeutic pathways. 2014;15(3):75-84.

14. Urbanus AT, van Keep M, Matser AA, et al. Is adding HCV screening to the antenatal national screening program in Amsterdam, the Netherlands, cost-effective? PLoS One. 2013;8(8):e70319.

15. Cipriano LE, Zaric GS, Holodniy M, Bendavid E, Owens DK, Brandeau ML. Cost effectiveness of screening strategies for early identification of $\mathrm{HIV}$ and $\mathrm{HCV}$ infection in injection drug users. PLoS One. 2012;7(9):e45176.

16. Sutton AJ, Edmunds WJ, Sweeting MJ, Gill ON. The cost-effectiveness of screening and treatment for hepatitis $\mathrm{C}$ in prisons in England and Wales: a cost-utility analysis. J Viral Hepat. 2008;15(11):797-808.

17. Eckman MH, Talal AH, Gordon SC, Schiff E, Sherman KE. Cost-effectiveness of screening for chronic hepatitis $\mathrm{C}$ infection in the United States. Clin Infect Dis. 2013;56(10):1382-1393.

18. Liu S, Cipriano LE, Holodniy M, Goldhaber-Fiebert JD. Cost-effectiveness analysis of risk-factor guided and birth-cohort screening for chronic hepatitis $\mathrm{C}$ infection in the United States. PLoS One. 2013;8(3):e58975.
19. Ruggeri M, Coretti S, Gasbarrini A, Cicchetti A. Economic assessment of an anti-HCV screening program in Italy. Value Health. 2013;16(6): 965-972.

20. Coffin PO, Scott JD, Golden MR, Sullivan SD. Cost-effectiveness and population outcomes of general population screening for hepatitis C. Clin Infect Dis. 2012;54(9):1259-1271.

21. McGarry LJ, Pawar VS, Panchmatia HR, et al. Economic model of a birth cohort screening program for hepatitis C virus. Hepatology. 2012; 55(5):1344-1355.

22. Nakamura J, Terajima K, Aoyagi Y, Akazawa K. Cost-effectiveness of the national screening program for hepatitis $\mathrm{C}$ virus in the general population and the high-risk groups. Tohoku J Exp Med. 2008;215(1): 33-42.

23. Tramarin A, Gennaro N, Compostella FA, Gallo C, Wendelaar Bonga LJ, Postma MJ. HCV screening to enable early treatment of hepatitis C: a mathematical model to analyse costs and outcomes in two populations. Curr Pharm Des. 2008;14(17):1655-1660.

24. Liu S, Schwarzinger M, Carrat F, Goldhaber-Fiebert JD. Cost effectiveness of fibrosis assessment prior to treatment for chronic hepatitis $\mathrm{C}$ patients. PLoS One. 2011;6(12):e26783.

25. Canavan C, Eisenburg J, Meng L, Corey K, Hur C. Ultrasound elastography for fibrosis surveillance is cost effective in patients with chronic hepatitis C virus in the UK. Dig Dis Sci. 2013;58(9):2691-2704.

\section{Publish your work in this journal}

Risk Management and Healthcare Policy is an international, peerreviewed, open access journal focusing on all aspects of public health, policy, and preventative measures to promote good health and improve morbidity and mortality in the population. The journal welcomes submitted papers covering original research, basic science, clinical \& epidemio-

\section{Dovepress}

logical studies, reviews and evaluations, guidelines, expert opinion and commentary, case reports and extended reports. The manuscript management system is completely online and includes a very quick and fair peerreview system, which is all easy to use. Visit http://www.dovepress.com/ testimonials.php to read real quotes from published authors. 